MIDPI

MOL2NET, International Conference Series on Multidisciplinary Sciences Insert the title of the workshop here

\title{
sciforum
}

\section{In Vitro and In Silico Study of Cytotoxicity of Metal Oxide Nanoparticles towards Escherichia coli}

\author{
SupratikKar (supratik.kar@icnanotox.org) ${ }^{l}$, Kavitha Pathakoti \\ (kavitha.pathakoti@jsums.edu) ${ }^{1,2}$,Danuta Leszczynska (danuta@icnanotox.org) ${ }^{1}$,Jerzy \\ Leszczynski (jerzy@icnanotox.org) ${ }^{I}$
}

\author{
${ }^{1}$ Interdisciplinary Center for Nanotoxicity, Department of Chemistry, Physics and Atmospheric \\ Sciences, Jackson State University, Jackson, MS-39217, USA \\ ${ }^{2}$ Department of Biology, Jackson State University, Jackson, MS-39217, USA
}

\begin{tabular}{|c|c|}
\hline $\begin{array}{c}\text { In silico study: Classification and } \\
\text { machine learning models }\end{array}$ & 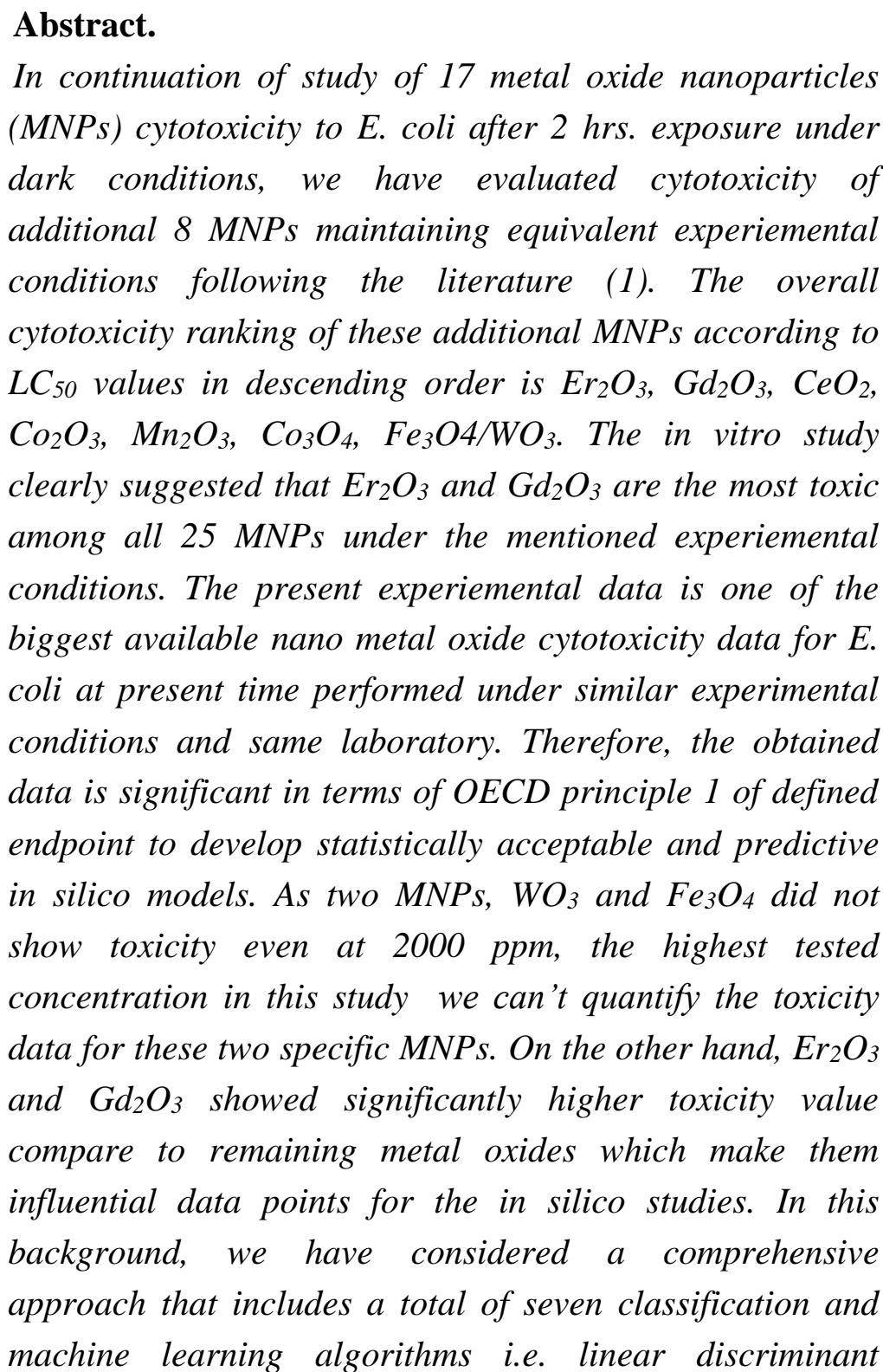 \\
\hline
\end{tabular}


analysis (LDA), nä̈ve bayes, multinomial logistic regression, Sequential minimal optimization (SMO), AdaBoost, J48 and random forest to model all 25 MNPs to understand and identify the major mechanism for such toxicities (2,3) without excluding any MNPs. To correlate the toxicity employing the in silico tools, we have employed $1^{s t}(4)$ and $2^{\text {nd }}$ (5) generation periodic table descriptors developed by us which can be computed in no time without any sophisticated computing facilities. Among the seven models, the LDA based model emerged as the best model considering goodness-of-fit and prediction capability checked on training $\left(N_{\text {Training }}=17\right)$ and test set $\left(N_{\text {Test }}=8\right)$. The electronegativity count of oxygen and the core environment of metal defined by the ratio of the number of core electrons to the number of valence electrons showed positive contributions towards toxicity. The identification of these molecular descriptors may be beneficial in explaining the mechanisms of nanotoxicity and for predicting the environmental risk associated with release of the MNPs. The developed models can be resourcefully employed for environmental risk assessment tools for the E. coli for any new/untested MNPs along with the influence in the future design and manufacture of safe nanomaterials.

\section{References}

1. T. Puzyn, B. Rasulev, A. Gajewicz, X. Hu, T.P. Dasari, A. Michalkova, H.M. Hwang, A. Toropov, D. Leszczynska, J. Leszczynski, Using nano-QSAR to predict the cytotoxicity of metal oxide nanoparticles, Nat. Nanotechnol. 2011, 6, 175-178.

2. Weka 3: Machine Learning Software in Java (https://www.cs.waikato.ac.nz/ml/weka/)

3. DTC Lab Software Tools available at https://sites.google.com/site/dtclabdc/

4. S. Kar, A. Gajewicz, T. Puzyn, K. Roy, J. Leszczynski, Periodic table-based descriptors to encode cytotoxicity profile of metal oxide nanoparticles: A mechanistic QSTR approach, Ecotoxicol. Environ. Saf. 2014, 107, 162-169.

5. P. De, S. Kar, K. Roy, J. Leszczynski, Second Generation Periodic Table Based Descriptors to Encode Toxicity of Metal Oxide Nanoparticles to Multiple Species: QSTR Modeling for Exploration of Toxicity Mechanisms. Environ. Sci.: Nano, 2018, 5, 2742-2760.

\section{Acknowledgement:}

Authors are thankful to the National Science Foundation (NSF/CREST HRD-1547754 and NSF/RISE HRD-1547836) for financial support. 